\title{
EDITOR'S PREFACE
}

IAU Colloquium No. 53 and the Fourth Annual Workshop on Cataclysmic Variables were co-sponsored by Comission 27 (Varlable Stars) and by Commission 35 (Stellar Structure). They also enjoyed the support of the American Astronomical Soclety. The meetings were purposely organized to bring together astronomers involved in the study of single white dwarfs and of white dwarfs in cataclysmic binary systems. The response was much greater than had been anticipated, especially in regard to the interest in white dwarfs in binaries, resulting in a rather crowded program. The original plan had been to devote Session I of the Colloquium to an overview of white dwarf evolution, Session II to EUV objects and hot white dwarfs, Session III to cool white dwarfs, Session IV to distributions of white dwarfs, Session.V to magnetIc white dwarfs and AM Her Stars, Session VI to oscillating white dwarfs, and Session VII to white dwarfs in close binaries. In addition, the two Workshop sessions were to be devoted to dwarf novae and to novae, respectively. To accomodate the many contributions dealing with white dwarfs in binaries, however, the topical nature of the Colloquium sessions had to be modified.

In compiling these Proceedings, we debated whether to rearrange the manuscripts by topics or to retain the papers in the order in which they were actually presented. We have chosen to do the latter. This has the consequence that papers on accreting white dwarfs and white dwarfs in binaries appear throughout, rather than being collected together in a single group. We have also retained the subdivision of the Proceedings into the different sessions, each session beginning with two Invited review papers followed by the shorter contributed papers. Contributions to the Workshop are here represented by abstracts only.

On behalf of the Organizing Committees, we should like to thank the participants in this Colloguium for a very informative meeting and for getting their manuscripts submitted in a timely way. Special thanks are also due to Mr. Don Parry of the University of Rochester Office of Special Events, who was largely responsible for the smooth running of the program, and to Mrs. Linda Van Vlifet who handled not only the correspondence but also the burden of editorial corrections.

Part of the time spent in editing this work was supported by the National Science Foundation through grant AST 78-27408 to the University of Rochester.

H.M. Van Horn

V. Weidemann 